\title{
The Antibacterial and Antibiofilm Activity of Telithromycin Against Enterococcus spp. Isolated From Patients in China
}

\author{
Yanpeng Xiong ${ }^{1 \dagger}$, Junwen Chen ${ }^{1+}$, Xiang Sun ${ }^{1+}$, Guangjian Xu' ${ }^{1}$, Peiyu $\mathrm{Li}^{1}$, Qiwen Deng ${ }^{1,2}$, \\ Zhijian Yu ${ }^{1,2}$, Zhong Chen ${ }^{1,2 *}$ and Jinxin Zheng ${ }^{1,2 *}$ \\ 1 Department of Infectious Diseases and Shenzhen Key Laboratory for Endogenous Infections, Shenzhen Nanshan People's \\ Hospital, Shenzhen University of School Medicine, Shenzhen, China, ${ }^{2}$ Quality Control Center of Hospital Infection \\ Management of Shenzhen, Shenzhen Nanshan People's Hospital of Guangdong Medical University, Shenzhen, China
}

\section{OPEN ACCESS}

Edited by:

Rodolfo García-Contreras, National Autonomous University

of Mexico, Mexico

Reviewed by:

Zhangya Pu,

Xiangya Hospital, Central South

University, China

Corina-Diana Ceapa,

National Autonomous University

of Mexico, Mexico

*Correspondence:

Zhong Chen

cchen17@fudan.edu.cn

Jinxin Zheng

jinxinzheng@fudan.edu.cn

${ }^{\dagger}$ These authors have contributed equally to this work

Specialty section:

This article was submitted to Antimicrobials, Resistance

and Chemotherapy,

a section of the journal

Frontiers in Microbiology

Received: 13 October 2020 Accepted: 14 December 2020

Published: 14 January 2021

Citation:

Xiong $Y$, Chen J, Sun X, XU G, Li P, Deng Q, Yu Z, Chen $Z$ and

Zheng J (2021) The Antibacterial and Antibiofilm Activity of Telithromycin Against Enterococcus spp. Isolated From Patients in China.

Front. Microbiol. 11:616797.

doi: 10.3389/fmicb.2020.616797
Telithromycin has been reported to possess robust in vitro antibacterial activity against many species of gram-positive bacteria, and telithromycin is also effective against Staphylococcus aureus biofilms. However, the in vitro antimicrobial susceptibility of telithromycin against clinical enterococci isolates in China is rarely reported and the impacts of telithromycin on the biofilm formation and eradication of enterococci remain elusive. Therefore, this study aimed to explore the inhibitory effects of telithromycin on planktonic cells and biofilms of Enterococcus strains. A total of 280 Enterococcus faecalis and 122 Enterococcus faecium isolates were collected from individual inpatients in China. The $50 \%$ minimum inhibitory concentration $\left(\mathrm{MIC}_{50}\right)$ values of telithromycin against the $E$. faecalis and $E$. faecium strains carrying erythromycin-resistant methylase (erm) genes such as the ermA, ermB, or ermC, were 2 and $4 \mu \mathrm{g} / \mathrm{mL}$, respectively. In addition, these isolates were typed using multilocus sequence typing (MLST) based on housekeeping genes. The predominant sequence types (STs) of E. faecalis were ST16, ST30, and ST179, and the main STs of E. faecium isolates were ST18, ST78, and ST80. Among these major STs, 87.1\% (135/158) of E. faecalis and 80.4\% (41/51) of $E$. faecium carried erm genes. Furthermore, at the subinhibitory concentrations (1/4 and $1 / 8 \times \mathrm{MIC}$ ) of telithromycin, the biofilm formation of $16 \mathrm{E}$. faecalis isolates were inhibited by approximately 35\%. Moreover, treatment with $8 \times \mathrm{MIC}$ of telithromycin or ampicillin led to an almost $40 \%$ reduction in the established biofilms of $E$. faecalis isolates, whereas vancomycin or linezolid with $8 \times \mathrm{MIC}$ had minimal effects. The combination of telithromycin and ampicillin resulted in an almost $70 \%$ reduction in the established biofilms of $E$. faecalis. In conclusion, these results revealed that telithromycin significantly decreased the planktonic cells of both $E$. faecalis and E. faecium. In addition, the data further demonstrated that telithromycin has the robust ability to inhibit $E$. faecalis biofilms and the combination of telithromycin and ampicillin improved antibiofilm activity. These in vitro antibacterial and antibiofilm activities suggest that telithromycin could be a potential candidate for the treatment of enterococcal infections.

Keywords: telithromycin, Enterococcus faecalis, Enterococcus faecium, erythromycin-resistance, MIC, MLST, biofilm 


\section{INTRODUCTION}

Enterococci are gram-positive cocci which are commonly found in the gastrointestinal tracts of nearly all land animals, including humans (Fiore et al., 2019). Although a core member of the microbiome, enterococci are capable of resulting in various infectious diseases, such as urinary tract infections, wound infections, intra-abdominal and pelvic regions infections, and bloodstream infections (Murray and Weinstock, 1999; Richards et al., 2000; Mohamed and Huang, 2007). Enterococci are now the third most common nosocomial pathogen. Statistics showed that enterococci caused almost 15\% of hospital-acquired infections in the United States between 2011 and 2014 (Weiner et al., 2016), an increase of 12\% from 2006 to 2007 (Hidron et al., 2008). In addition, enterococci are also responsible for 5-20\% of cases of infective endocarditis (Megran, 1992). Enterococcus faecalis, the most common species of Enterococcus in the clinical setting, causes 85 to $90 \%$ of human enterococcal infections, while Enterococcus faecium is responsible for 5 to $10 \%$ of the remainder (Jett et al., 1994; Jones et al., 2004). Because E. faecalis and E. faecium usually carry a range of intrinsic and acquired resistance genes, these Enterococcus strains are frequently resistant to many commonly used antibiotics, such as glycopeptides (vancomycin and teicoplanin), betalactams (ampicillin, penicillin), aminoglycoside (gentamicin or streptomycin), and macrolides (van Harten et al., 2017; Ch'ng et al., 2019). Of note, the widespread emergence of vancomycinresistant enterococci (VRE) has caused further concern due to the high mortality rate (Eliopoulos, 1997). In 2013, almost 70\% of clinical E. faecium isolates in the United States displayed vancomycin resistance, while this was up to $20 \%$ in Europe (Mendes et al., 2016). In contrast, E. faecalis isolates are less frequently resistant to vancomycin (<10\%) (Sievert et al., 2013).

Besides antibiotic resistance, enterococci are also known for their ability to form biofilms which is a population of cells growing on a surface and surrounded by a matrix of macromolecules like polysaccharides, proteins, lipids, and extracellular DNA (Jakubovics and Burgess, 2015). The term biofilm was introduced into medicine in 1982 by Costerton as Staphylococcus aureus biofilms were observed on a cardiac pacemaker lead (Marrie et al., 1982). Bacteria that are not innately resistant to antibiotics can also become resistant by forming persistent biofilms that lead to chronic infections (Lebeaux et al., 2014). In today's healthcare environment, the diversity of biofilm-associated infections has risen with time, it is suggested that biofilms are present in more than $65 \%$ of all bacterial infections (Costerton et al., 1999; Lewis, 2001). Especially, due to a sharp increase in the number of patients receiving implanted medical devices in recent years, the rates of infection are $40 \%$ for ventricular assist devices, $10 \%$ for ventricular shunts, and $4 \%$ for mechanical heart valves, pacemakers, and defibrillators (Darouiche, 2004).

Abbreviations: AMP, ampicillin; CIP, ciprofloxacin; DOX, doxycycline; ERY, erythromycin; LZD, linezolid; MICs, minimum inhibitory concentrations; MIN, minocycline; MLST, multilocus sequence type; NIT, nitrofurantoin; PCR, polymerase chain reaction; STs, sequence types; TEC, tetracycline; TED, tedizolid; TEL, telithromycin; TET, tetracycline; VAN, vancomycin.
Biofilms serve as a new nidus for bacterial dissemination and as a reservoir for antimicrobials resistant genes. Additionally, biofilms protect bacteria from detergent solutions, antimicrobial agents, environmental stress, and effectively make bacteria 10 to 1000-fold more resistant to antibiotic treatment, making their eradication extremely difficult (Lewis, 2001). Therefore, biofilm infections are becoming increasingly difficult to effectively treat due to the decreasing efficacy of antibiotics (Paganelli et al., 2012; van Harten et al., 2017). Enterococci frequently cause biofilm-associated infections such as catheter-related bloodstream infections, urinary tract infections, and infective endocarditis (Arias and Murray, 2012). Among Enterococcus species, E. faecalis isolates usually have a higher capacity of producing biofilms than E. faecium isolates and the prevalence of $E$. faecalis biofilms varies in different regions (Mohamed and Huang, 2007). For example, in Sardinia, biofilm production was identified among $87 \%$ of E. faecalis clinical isolates and $16 \%$ of E. faecium clinical isolates (Duprè et al., 2003). In Rome, $80 \%$ of E. faecalis and $48 \%$ of E. faecium isolates from infected patients were able to form biofilms (Baldassarri et al., 2001). Other study showed similar results and indicated that E. faecalis (95\%) isolates produced biofilms more often than E. faecium (29\%) (Di Rosa et al., 2006). In contrast with planktonic cells, biofilm-embedded enterococci are involved in multidrug resistance and may even be untreatable with conventional antibiotics (Lewis, 2001). Therefore, there is an urgent need to identify novel treatments for enterococcal infections.

Telithromycin (HMR 3647), a semi-synthetic derivative of erythromycin, belongs to a new chemical class of antibiotics called ketolides that have been added to the macrolidelincosamide-streptogramin class of antibiotics (Douthwaite and Champney, 2001). Telithromycin has been approved by the U.S. Food and Drug Administration (FDA) to treat several infectious diseases, such as community-acquired pneumonia, acute exacerbations of chronic bronchitis, and acute maxillary sinusitis (Nguyen and Chung, 2005). The antimicrobial action of telithromycin, and probably that of the other 14-memberring macrolides, is compounded by binding to $23 \mathrm{~S}$ rRNA and blocking protein synthesis at the early stages (Menninger, 1995). Macrolides resistance is dominantly explained by the prevalence of $\mathrm{erm}$ genes in a wide spectrum of gram-positive bacteria (Westh et al., 1995). erm gene classes can encode a series of methyltransferase that specifically methylate the N-6 position of adenosine 2058 (A2058) or neighboring nucleotides in domain $\mathrm{V}$ of $23 \mathrm{~S}$ rRNA within the large ribosomal subunit (Weisblum, 1995; Vester and Douthwaite, 2001), this prevents interaction with macrolides. In addition, erythromycin-resistant enterococci often exhibit macrolide-lincosamide-streptogramin $\mathrm{B}$ (MLSB) antibiotic resistance phenotypes due to the presence of erm genes. However, compared with MLSB antibiotics like erythromycin, telithromycin has an additional target site at position A752 in domain II of $23 \mathrm{~S}$ rRNA. U747 methylation promotes G748 methylation, resulting in the increased binding of telithromycin to ribosomes and enhanced telithromycin susceptibility (Shoji et al., 2015). Telithromycin has been found to conquer erm-mediated resistance in S. pneumonia 
and S. aureus (Van Laethem and Sternon, 2003; Sun et al., 2018), and it also showed less efficacy against erythromycinresistant Enterococcus isolates from different regions (TEL, MIC $\geq 4 \mu \mathrm{g} / \mathrm{mL}$ ) (Singh et al., 2000; Min et al., 2011). However, the antimicrobial impacts of telithromycin on Enterococcus carrying erm genes remain less clear in China. Moreover, a previous study reported that telithromycin exhibited effective antibiofilm activity against $S$. aureus in vitro (Zheng et al., 2020). Therefore, the effect of telithromycin on Enterococcus biofilms should be further investigated.

In this study, the in vitro antibacterial activity of telithromycin was tested against enterococcal clinical isolates from inpatients in Shenzhen Nanshan People's Hospital, and then the antibacterial activity of telithromycin was further compared with that of other antimicrobials. In addition, the multilocus sequence types (MLSTs) and erm genes expression in enterococci were detected by PCR assay. Moreover, the effect of telithromycin on the biofilms of $E$. faecalis isolates was further explored.

\section{MATERIALS AND METHODS}

\section{Bacterial Isolates and Growth Conditions}

A total of 280 E. faecalis and 122 E. faecium strains were isolated from individual patients at Shenzhen Nanshan People's Hospital from 2011 to 2015. Bacterial isolates were determined by standard methods using a VITEK 2 system (Biomérieux, Marcy l'Etoile, France). E. faecalis ATCC 29212 and OG1RF (ATCC 47077) were tested as quality control strains. The isolates were cultured overnight in tryptic soy broth (TSB) (Oxoid, Basingstoke, United Kingdom) at $37^{\circ} \mathrm{C}$ with a shaker at $220 \mathrm{rpm}$. Telithromycin (TEL), erythromycin (ERY), ampicillin (AMP), vancomycin (VAN), tetracycline (TET), doxycycline (DOX), minocycline (MIN), ciprofloxacin (CIP), nitrofurantoin (NIT), linezolid (LZD), tedizolid (TED), and tetracycline (TEC) were purchased from MCE (Princeton, NJ, United States).

\section{Antimicrobials Susceptibility Testing}

Antimicrobial susceptibilities of Enterococcus to several clinical antibiotics, including TEL, ERY, AMP, VAN, TET, DOX, MIN, CIP, NIT, LZD, TED, and TEC were tested with the VITEK 2 system. The MICs of AMP, VAN, TEL, and ERY were determined by the broth macrodilution method in cationadjusted Mueller-Hinton broth (CAMHB) according to the 2019 Clinical and Laboratory Standards Institute (CLSI) guidelines ${ }^{1}$. The isolates were cultured overnight in TSB at $37^{\circ} \mathrm{C}$ with a shaker at $220 \mathrm{rpm}$, then the strains were diluted at 1:200 [2.0$3.0 \times 10^{7}$ colony-forming units (CFU) $\mathrm{ml}^{-1}$ ], and inoculated into 96 polystyrene microtiter plates with $200 \mu \mathrm{L}$ of CAMHB containing the indicated concentrations of antibiotics. As the MIC breakpoint of telithromycin against enterococci has not been established, the MIC value of telithromycin against $S$. aureus $(\leq 1,2, \geq 4 \mu \mathrm{g} / \mathrm{mL})$ was based on the 2019 CLSI guidelines. Four MIC levels were thus employed for telithromycin in the

\footnotetext{
${ }^{1}$ https://eucast.org/clinical_breakpoints/
}

antimicrobial susceptibility analysis $(\leq 1,2,4, \geq 8 \mu \mathrm{g} / \mathrm{mL})$. The used concentrations of indicated antibiotics are given in the figure legends and Supplementary Table 4. All experiments were performed at least three times.

\section{Detection of ERY Resistance Genes}

DNA was extracted from all clinical enterococcal isolates with lysis buffer as templates for PCR according to the manufacturer's instructions (Takara Bio Inc., Japan). As described previously (Bai et al., 2018), PCR analysis was performed to detect ermA, ermB, and erm $C$ genes, and the primers used for PCR amplification were as follows:

ermA: sense: $5^{\prime}$ - TCTAAAAAGCATGTAAAAGAAA- $3^{\prime}$ and antisense: $5^{\prime}$ - CGATACTTTTTGTAGTCCTTC-3'; ermB: sense: $5^{\prime}$-CCGTTTACGAAATTGGAACAGGTAAAGGGC-3' and antisense: $5^{\prime}$-GAATCGAGACTTGAGTGTGC-3'; ermC: sense: $5^{\prime}$-GCTAATATTGTTTAAATCGTCAATTCC- $3^{\prime}$ and antisense: $5^{\prime}$ - GGATCAGGAAAAGGACATTTTAC - $3^{\prime}$.

\section{Multilocus Sequence Type}

The genotypes of the enterococcal isolates were analyzed by MLST. Seven pairs of housekeeping genes: $g d h, g y d, p s t S, g k i$, aroE, $x p t$, and yqiL for E. faecalis, and atpA, ddl, gdh, purK, gyd, $p s t S$, and $a d k$ for E. faecium were amplified by PCR. As previously reported (Zheng et al., 2017), the purified PCR products were sequenced, and the results were submitted to the MLST database ${ }^{2}$ for comparison, and the sequence types (STs) of enterococci were determined. The primers used for PCR amplification are listed in Supplementary Tables 5, 6.

\section{Inhibition and Eradication of E. faecalis Biofilms}

A detailed protocol has been previously reported (Zheng et al., 2020). As for the inhibition experiments, E. faecalis isolates were cultured overnight in TSB at $37^{\circ} \mathrm{C}$ with a shaker at $220 \mathrm{rpm}$, then the strains were diluted at 1:200 [2.0-3.0 $\times 10^{7}$ colony-forming units (CFU) $\mathrm{ml}^{-1}$ ], and inoculated into 96 polystyrene microtiter plates with $200 \mu \mathrm{L}$ of TSBG (TSB with $0.5 \%$ glucose) containing the indicated concentration of antibiotics. TSBG without antimicrobials was used as an untreated control. After incubation for $24 \mathrm{~h}$, the biofilm biomasses were washed three times with $\mathrm{ddH}_{2} \mathrm{O}$ before and after crystal violet staining, the stained biofilms were detected by optical density $\left(\mathrm{OD}_{570}\right)$. Solithromycin was used as a positive control (Wang et al., 2020). As for the eradication assays of the established biofilms, E. faecalis isolates were cultivated in tryptic TSB medium at $37^{\circ} \mathrm{C}$ for $24 \mathrm{~h}$ to form matured biofilms, then they were treated with antimicrobials $(8 \times$ MIC) for $48 \mathrm{~h}$ with the medium replaced daily. TSBG without antimicrobials was used as an untreated control. Biofilm biomasses were stained and then detected. The used concentrations of indicated antibiotics are given in the figure legends and Supplementary Table 4. All data are representative of three independent experiments.

\footnotetext{
${ }^{2}$ http://efaecalis.mlst.net/ and http://efaecium.mlst.net/
} 


\section{Detecting the Adherent Cells in the Established Biofilms}

The adherent cells in the established biofilms of E. faecalis were identified by the CFU numbers as described previously (Zheng et al., 2019). Briefly, the E. faecalis isolates were inoculated into 24 polystyrene microtiter plates with TSBG and formed mature biofilms after $24 \mathrm{~h}$ of static incubation. The supernatant was discarded and plates were washed, fresh TSBG containing antimicrobials was added, and TSBG without antimicrobials was used as an untreated control. After $48 \mathrm{~h}$ of static incubation with the medium being replaced daily, the supernatant was discarded and the remaining adherent cells in the established biofilms were collected by scratching the wall of the wells with a flat end toothpick. Finally, the bacteria were centrifuged and the numbers of CFU were determined. The used concentrations of indicated antibiotics were given in the figure legends and Supplementary Table 4. All data are representative of three independent experiments.

\section{Time-Kill Curve Assay}

Two E. faecalis 16C3 and 16C6 isolates were cultured in TSB at $37^{\circ} \mathrm{C}$ for $16 \mathrm{~h}$, then diluted 200 times with TSB and antibiotics were added to make the final concentrations at $4 \times$ MIC. A colony count was performed after $0,1,3$, and $24 \mathrm{~h}$. Data are representative of three independent experiments.

\section{Statistical Analysis}

The SPSS software (version 19.0) and GraphPad Prism software (version 5.0) were used for statistical analysis. For multiple comparisons, one-way analysis of variance (ANOVA) and a post hoc-Dunnett test were applied to analyze the data. All experiments were repeated at least three times. Sample size, $\mathrm{n}$, for each experiment is given in the figure legends. Results are shown as mean \pm SEM. Value differences were considered significant when ${ }^{*} p<0.05$ (not significant $p>0.05,{ }^{* *} p<0.01$, $* * * p<0.001)$.

\section{RESULTS}

\section{In vitro Antibacterial Activity of Telithromycin Against Enterococci Isolates}

Enterococcus faecalis isolates $(n=280)$ and E. faecium isolates $(n=122)$ were retrospectively collected from different clinical specimens in China, including urine, wound secretions, blood, bile, phlegm, and other sources (Supplementary Figures 1A,B). The in vitro antibacterial activity of telithromycin against clinical isolates of E. faecalis and E. faecium are summarized in Tables 1, 2. As expected, these clinical isolates of enterococci showed a high frequency of resistance to erythromycin (ERY, MIC $\geq 8 \mu \mathrm{g} / \mathrm{mL}$ ) and some commonly used tetracycline antibiotics, including tetracycline (TET, MIC $\geq 16 \mu \mathrm{g} / \mathrm{mL}$ ), doxycycline (DOX, MIC $\geq 16 \mu \mathrm{g} / \mathrm{mL}$ ), and minocycline (MIN, $\mathrm{MIC} \geq 16 \mu \mathrm{g} / \mathrm{mL})$, whereas they remained highly susceptible to vancomycin (VAN, MIC $\leq 4 \mu \mathrm{g} / \mathrm{mL}$ ), nitrofurantoin (NIT, $\mathrm{MIC} \leq 32 \mu \mathrm{g} / \mathrm{mL}$ ), and linezolid (LZD, MIC $\leq 2 \mu \mathrm{g} / \mathrm{mL}$ ).

In addition, as shown in Tables 1, 2, both E. faecalis and E. faecium isolates had a high $\mathrm{MIC}_{50} / \mathrm{MIC}_{90}$ (the $\mathrm{MIC}$ values for $50 \%$ or $90 \%$ of bacterial growth inhibition) of ERY $(>256 />256 \mu \mathrm{g} / \mathrm{mL})$. However, the $\mathrm{MIC}_{50} / \mathrm{MIC}_{90}$ values of telithromycin against the E. faecalis and E. faecium strains were $2 / 8 \mu \mathrm{g} / \mathrm{mL}$ and $4 / 8 \mu \mathrm{g} / \mathrm{mL}$, respectively. Moreover, $75.7 \%(212 / 280)$ of $E$. faecalis isolates were shown with ERY $\mathrm{MIC} \geq 8 \mu \mathrm{g} / \mathrm{mL}$, whereas only $11.8 \%$ (33/280) of E. faecalis isolates were shown with telithromycin $\mathrm{MIC} \geq 8 \mu \mathrm{g} / \mathrm{mL}$ (Table 1). Similarly, among 122 E. faecium isolates, $85.2 \%(104 / 122)$ of strains had a high resistance to ERY (MIC $\geq 8 \mu \mathrm{g} / \mathrm{mL})$, whereas $40 \%$ (49/122) of strains were shown with telithromycin MIC $\geq 8 \mu \mathrm{g} / \mathrm{mL}$ (Table 2), suggesting that Enterococcus isolates were more susceptible to telithromycin than ERY. Interestingly, it was found that there was a high susceptibility rate of $99.6 \%(274 / 275)$ for E. faecalis isolates toward ampicillin (MIC $\leq 8 \mu \mathrm{g} / \mathrm{mL}$ ), whereas there was a high resistant rate of $87.5 \%(98 / 112)$ for E. faecium isolates to ampicillin ( $\mathrm{MIC} \geq 16 \mu \mathrm{g} / \mathrm{mL}$ ). These results indicate that the antibacterial activity of telithromycin against Enterococcus was better than that of ERY.

\section{Telithromycin Against the Enterococcus Clinical Isolates Harboring erm Genes}

Next, this study further examined the effects of E. faecalis and E. faecium isolates carrying erm genes on the sensitivity of telithromycin. At first, the presence of ermA, ermB, or erm $C$ in Enterococcus isolates was detected by PCR assays. As shown in Table 3, the rates of E. faecalis strains harboring ermA and $\mathrm{erm} B$ genes were 3.9 and $67.1 \%$, respectively. However, there were no ermC-positive E. faecalis isolates. Additionally, among 122 E. faecium isolates, the rates of E. faecium isolates carrying ermA, $\mathrm{erm} B$, and erm $C$ gene were 9.8, 32.8, and 45.9\%, respectively (Table 4). Furthermore, telithromycin $\mathrm{MIC}_{50} / \mathrm{MIC}_{90}$ values of erm $A$ and ermB-positive E. faecalis strains or E. faecium strains both were $4 / 8 \mu \mathrm{g} / \mathrm{mL}$, and erm $C$-positive E. faecium strains had a telithromycin $\mathrm{MIC}_{50} / \mathrm{MIC}_{90}$ of $8 / 8 \mu \mathrm{g} / \mathrm{mL}$ (Tables 3, 4). As mentioned, the $\mathrm{MIC}_{50} / \mathrm{MIC}_{90}$ values of telithromycin against E. faecalis and E. faecium strains were $2 / 8$ and $4 / 8 \mu \mathrm{g} / \mathrm{mL}$, respectively (Tables 1,2 ), suggesting that the presence of erm genes slight impacted telithromycin susceptibility in the Enterococcus isolates.

\section{Relationship Between Telithromycin MICs Distribution and ST Clonality}

Subsequently, MLST was performed to determine the ST distribution of E. faecalis and E. faecium isolates. As shown in Supplementary Tables 1, 2, ST16, ST30, and ST179 were the predominant STs in 44 STs detected from E. faecalis isolates. In total, 25 STs were identified in E. faecium isolates, the main STs were ST18, ST78, and ST80. In addition, the relationships between telithromycin and ERY MICs distributions in the predominant ST isolates are shown in Tables 5, 6. The data indicated that E. faecalis with telithromycin $\mathrm{MIC} \leq 1,2,4$, 
TABLE 1 | In vitro antibacterial activity of TEL compared with that of various antibiotics against $E$. faecalis isolates.

\begin{tabular}{|c|c|c|c|c|c|c|c|c|c|c|c|c|c|c|}
\hline \multirow[t]{2}{*}{ Antibiotic } & \multirow{2}{*}{$\begin{array}{c}\text { No. } \\
\text { isolates }\end{array}$} & \multirow{2}{*}{$\begin{array}{c}\text { Resistance } \\
\text { rate (\%) }\end{array}$} & \multirow{2}{*}{$\begin{array}{l}\text { MIC }(\mu \mathrm{g} / \mathrm{mL}) \\
\text { breakpoints }\end{array}$} & \multirow[t]{2}{*}{ NO. } & \multicolumn{6}{|c|}{ TEL MIC $(\mu \mathrm{g} / \mathrm{mL})$} & \multicolumn{4}{|c|}{ ERY MIC $(\mu \mathrm{g} / \mathrm{mL})$} \\
\hline & & & & & $\leq 0.5$ & 1 & 2 & 4 & $\geq 8$ & $\mathrm{MIC}_{50} / \mathrm{MIC}_{90}$ & $\leq 0.5$ & $1-4$ & $\geq 8$ & $\mathrm{MIC}_{50} / \mathrm{MIC}_{90}$ \\
\hline Total & 280 & - & - & - & 116 & 7 & 27 & 97 & 33 & $2 / 8$ & 10 & 58 & 212 & $>256 />256$ \\
\hline \multirow[t]{2}{*}{ Ampicillin } & 275 & 0.4 & $\leq 8$ & 274 & 130 & 6 & 26 & 83 & 29 & $2 / 8$ & 8 & 55 & 211 & $>256 />256$ \\
\hline & & & $\geq 16$ & 1 & 0 & 0 & 0 & 1 & 0 & $4 / 4$ & 0 & 0 & 1 & - \\
\hline \multirow[t]{3}{*}{ Vancomycin } & 280 & 0 & $\leq 4$ & 278 & 115 & 7 & 27 & 96 & 33 & $2 / 8$ & 10 & 58 & 210 & $>256 />256$ \\
\hline & & & $8-16$ & 2 & 1 & 0 & 0 & 1 & 0 & $0.125 / 4$ & 0 & 0 & 2 & $>256 />256$ \\
\hline & & & $\geq 32$ & 0 & 0 & 0 & 0 & 0 & 0 & - & 0 & 0 & 0 & - \\
\hline \multirow[t]{3}{*}{ Tetracycline } & 276 & 83.7 & $\leq 4$ & 39 & 33 & 3 & 1 & 2 & 0 & $0.06 / 1$ & 7 & 17 & 15 & $4 />256$ \\
\hline & & & 8 & 6 & 3 & 0 & 0 & 2 & 1 & $0.06 / 4$ & 1 & 1 & 4 & $128 />256$ \\
\hline & & & $\geq 16$ & 231 & 79 & 4 & 25 & 91 & 32 & $4 / 8$ & 2 & 39 & 190 & $>256 />256$ \\
\hline \multirow[t]{3}{*}{ Doxycycline } & 280 & 78.9 & $\leq 4$ & 40 & 36 & 1 & 2 & 1 & 0 & $0.06 / 0.125$ & 7 & 19 & 14 & $2 />256$ \\
\hline & & & 8 & 19 & 7 & 2 & 3 & 6 & 1 & $2 / 4$ & 1 & 2 & 16 & $128 />256$ \\
\hline & & & $\geq 16$ & 221 & 73 & 4 & 22 & 90 & 32 & $4 / 8$ & 2 & 37 & 182 & $>256 />256$ \\
\hline \multirow[t]{3}{*}{ Minocycline } & 280 & 73.2 & $\leq 4$ & 43 & 37 & 2 & 2 & 2 & 0 & $0.06 / 2$ & 7 & 21 & 15 & $2 />256$ \\
\hline & & & 8 & 32 & 11 & 0 & 9 & 9 & 3 & $2 / 4$ & 1 & 5 & 26 & $>256 />256$ \\
\hline & & & $\geq 16$ & 205 & 68 & 5 & 16 & 86 & 30 & $4 / 8$ & 2 & 32 & 171 & $>256 />256$ \\
\hline \multirow[t]{3}{*}{ Ciprofloxacin } & 252 & 26.6 & $\leq 1$ & 151 & 68 & 3 & 11 & 51 & 18 & $2 / 8$ & 5 & 25 & 121 & $>256 />256$ \\
\hline & & & 2 & 34 & 22 & 1 & 2 & 8 & 1 & $0.125 / 4$ & 1 & 12 & 21 & $128 />256$ \\
\hline & & & $\geq 4$ & 67 & 24 & 3 & 14 & 20 & 6 & $2 / 8$ & 2 & 12 & 53 & $>256 />256$ \\
\hline \multirow[t]{3}{*}{ Nitrofurantoin } & 254 & 1.2 & $\leq 32$ & 247 & 102 & 6 & 26 & 84 & 29 & $2 / 8$ & 9 & 52 & 186 & $>256 />256$ \\
\hline & & & 64 & 4 & 2 & 0 & 0 & 2 & 0 & $0.5 / 4$ & 0 & 0 & 4 & $>256 />256$ \\
\hline & & & $\geq 128$ & 3 & 3 & 0 & 0 & 0 & 0 & $0.03 / 0.25$ & 0 & 2 & 1 & $4 />128$ \\
\hline \multirow[t]{3}{*}{ Linezolid } & 280 & 5.4 & $\leq 2$ & 214 & 87 & 6 & 21 & 75 & 25 & $2 / 8$ & 9 & 44 & 161 & $>256 />256$ \\
\hline & & & 4 & 51 & 23 & 1 & 6 & 17 & 4 & $2 / 4$ & 1 & 11 & 39 & $>256 />256$ \\
\hline & & & $\geq 8$ & 15 & 6 & 0 & 0 & 5 & 4 & $4 / 8$ & 0 & 3 & 12 & $>256 />256$ \\
\hline
\end{tabular}

MIC, minimum inhibitory concentration; TEL, telithromycin; ERY, erythromycin; MIC $50 / M I C_{90}$, the MIC values for $50 \%$ or $90 \%$ of bacterial growth inhibition.

$\geq 8 \mu \mathrm{g} / \mathrm{mL}$ accounted for $26.6 \%$ (42/158), 9.5\% (15/158), $47.5 \%$ (75/158), $16.4 \%(26 / 158)$ and that the rates of $E$. faecium isolates with telithromycin $\mathrm{MIC} \leq 1,2,4, \geq 8 \mu \mathrm{g} / \mathrm{mL}$ were $15.7 \%$ (8/51), 2\% (1/51), 23.5\% (12/51), 58.8\% (30/51), respectively. However, the ERY-resistant rates of E. faecalis and E. faecium isolates with the top three STs reached 98.7\% (156/158) and 94.1\% (48/51), respectively. Moreover, among the predominant STs isolates, $87.1 \%(135 / 158)$ of E. faecalis and $80.4 \%(41 / 51)$ of E. faecium were shown alongside the erm genes carriage (Tables 5, 6), demonstrating clonal clustering toward these predominant STs.

\section{The Effects of Telithromycin Against Biofilm Formation and Eradication of E. faecalis Clinical Isolates}

Many studies have demonstrated that E. faecalis isolates have a higher capacity of producing biofilms than E. faecium (Duprè et al., 2003; Sandoe et al., 2003; Zheng et al., 2017), thus E. faecalis isolates are usually chosen for biofilm analysis. Among our 280 E. faecalis isolates, 16 E. faecalis isolates showed a higher biofilm-forming ability, these specific 16 strains were thus tested for biofilm formation. The isolation sites of the $16 \mathrm{E}$. faecalis strains are listed in Supplementary Table 3. The inhibitory effect of telithromycin on the biofilm formation of these 16 E. faecalis isolates was determined by crystal violet staining.
The MIC values of AMP, VAN, LZD, and telithromycin against these isolates are listed in Supplementary Table 4. As shown in Figure 1, 1/2 $\times$ MIC, 1/4 $\times$ MIC, or 1/8 $\times$ MIC of telithromycin could inhibit the biofilm formation of the $16 \mathrm{E}$. faecalis isolates.

Finally, to dissect the effect of telithromycin on the established biofilms of E. faecalis isolates. Here, eight specific E. faecalis isolates were chosen for analysis by crystal violet staining. As shown in Figures 2A,B, the established biofilms of E. faecalis reduced by almost $40 \%$ after treatment with $8 \times$ MIC of telithromycin or ampicillin, whereas $8 \times$ MIC of vancomycin or linezolid only showed slight effects. Due to the high susceptibility rate of the E. faecalis isolates toward ampicillin (Table 1), the effects of telithromycin combined with ampicillin on the established biofilms of these eight E. faecalis isolates were further evaluated. The data showed that the combination of telithromycin and ampicillin resulted in an approximate $70 \%$ reduction in the established biofilms than with telithromycin or ampicillin alone (Figures 2C,D). Additionally, a colonyforming unit (CFU) assay was performed to quantify the viable cells of the established biofilms. Consistently, the data further confirmed that telithromycin combined with ampicillin could kill more than $70 \%$ of adherent cells in the established biofilms than telithromycin or ampicillin alone (Figures 2E,F). Therefore, these results suggest that the combination of telithromycin and ampicillin is an effective way to reduce the established biofilms in the E. faecalis isolates. 
TABLE 2 | In vitro antibacterial activity of TEL compared with that of various antibiotics against E. faecium isolates.

\begin{tabular}{|c|c|c|c|c|c|c|c|c|c|c|c|c|c|c|}
\hline \multirow[t]{2}{*}{ Antibiotic } & \multirow{2}{*}{$\begin{array}{c}\text { No. } \\
\text { isolates }\end{array}$} & \multirow{2}{*}{$\begin{array}{c}\text { Resistance } \\
\text { rate (\%) }\end{array}$} & \multirow{2}{*}{$\begin{array}{l}\text { MIC }(\mu \mathrm{g} / \mathrm{mL}) \\
\text { breakpoints }\end{array}$} & \multirow[t]{2}{*}{ No. } & \multicolumn{6}{|c|}{ TEL MIC ( $\mu \mathrm{g} / \mathrm{mL})$} & \multicolumn{4}{|c|}{ ERY MIC $(\mu \mathrm{g} / \mathrm{mL})$} \\
\hline & & & & & $\leq 0.5$ & 1 & 2 & 4 & $\geq 8$ & $\mathrm{MIC}_{50} / \mathrm{MIC}_{90}$ & $\leq 0.5$ & $1-4$ & $\geq 8$ & $\mathrm{MIC}_{50} / \mathrm{MIC}_{90}$ \\
\hline Total & 122 & - & - & - & 25 & 2 & 12 & 34 & 49 & $4 / 8$ & 6 & 12 & 104 & $>256 />256$ \\
\hline \multirow[t]{2}{*}{ Ampicillin } & 112 & 87.5 & $\leq 8$ & 14 & 11 & 0 & 0 & 3 & 0 & $0.06 / 4$ & 1 & 6 & 7 & $8 />128$ \\
\hline & & & $\geq 16$ & 98 & 13 & 2 & 12 & 29 & 42 & $4 / 8$ & 5 & 6 & 87 & $>128 />256$ \\
\hline \multirow[t]{3}{*}{ Vancomycin } & 120 & 0.0 & $\leq 4$ & 116 & 23 & 2 & 12 & 31 & 48 & $4 / 8$ & 6 & 11 & 99 & $128 />256$ \\
\hline & & & $8-16$ & 4 & 2 & 0 & 0 & 2 & 0 & $0.06 / 4$ & 0 & 1 & 3 & $8 />256$ \\
\hline & & & $\geq 32$ & 0 & 0 & 0 & 0 & 0 & 0 & - & 0 & 0 & 0 & - \\
\hline \multirow[t]{3}{*}{ Teicoplanin } & 115 & 0.9 & $\leq 8$ & 114 & 24 & 2 & 12 & 33 & 43 & $4 / 8$ & 6 & 12 & 96 & $>128 />256$ \\
\hline & & & 16 & 0 & 0 & 0 & 0 & 0 & 0 & - & 0 & 0 & 0 & - \\
\hline & & & $\geq 32$ & 1 & 0 & 0 & 0 & 0 & 1 & 8 & 0 & 0 & 1 & $>256 />256$ \\
\hline \multirow[t]{3}{*}{ Tetracycline } & 119 & 46.2 & $\leq 4$ & 45 & 11 & 1 & 1 & 16 & 16 & $4 / 8$ & 1 & 6 & 38 & $>128 />256$ \\
\hline & & & 8 & 19 & 3 & 0 & 1 & 3 & 12 & $8 / 8$ & 2 & 0 & 17 & $>256 />256$ \\
\hline & & & $\geq 16$ & 55 & 10 & 1 & 10 & 14 & 20 & $4 / 8$ & 3 & 5 & 47 & $>256 />256$ \\
\hline \multirow[t]{3}{*}{ Doxycycline } & 120 & 38.3 & $\leq 4$ & 62 & 15 & 1 & 4 & 22 & 20 & $4 / 8$ & 4 & 8 & 50 & $>128 />256$ \\
\hline & & & 8 & 12 & 1 & 0 & 4 & 1 & 6 & $4 / 8$ & 0 & 0 & 12 & $>256 />256$ \\
\hline & & & $\geq 16$ & 46 & 9 & 1 & 4 & 8 & 24 & $8 / 8$ & 2 & 4 & 40 & $>128 />256$ \\
\hline \multirow[t]{3}{*}{ Minocycline } & 120 & 27.5 & $\leq 4$ & 66 & 16 & 1 & 4 & 22 & 23 & $4 / 8$ & 4 & 8 & 54 & $>128 />256$ \\
\hline & & & 8 & 21 & 3 & 1 & 6 & 4 & 7 & $2 / 8$ & 1 & 1 & 19 & $>256 />256$ \\
\hline & & & $\geq 16$ & 33 & 6 & 0 & 2 & 7 & 18 & $8 / 8$ & 1 & 3 & 29 & $>128 />256$ \\
\hline \multirow[t]{3}{*}{ Ciprofloxacin } & 113 & 9.7 & $\leq 1$ & 29 & 10 & 1 & 7 & 8 & 3 & $2 / 4$ & 1 & 5 & 23 & $>256 />256$ \\
\hline & & & 2 & 4 & 1 & 0 & 0 & 1 & 2 & $4 / 8$ & 1 & 0 & 3 & $>256 />256$ \\
\hline & & & $\geq 4$ & 80 & 13 & 1 & 5 & 24 & 37 & $4 / 8$ & 3 & 7 & 70 & $>128 />256$ \\
\hline \multirow[t]{3}{*}{ Nitrofurantoin } & 114 & 57.0 & $\leq 32$ & 11 & 2 & 0 & 3 & 2 & 4 & $4 / 8$ & 1 & 1 & 9 & $>256 />256$ \\
\hline & & & 64 & 38 & 6 & 1 & 5 & 12 & 14 & $4 / 8$ & 1 & 4 & 33 & $>256 />256$ \\
\hline & & & $\geq 128$ & 65 & 16 & 1 & 4 & 18 & 26 & $4 / 8$ & 4 & 7 & 54 & $>128 />256$ \\
\hline \multirow[t]{3}{*}{ Linezolid } & 122 & 2.4 & $\leq 2$ & 116 & 22 & 2 & 12 & 32 & 48 & $4 / 8$ & 6 & 10 & 100 & $>128 />256$ \\
\hline & & & 4 & 3 & 2 & 0 & 0 & 1 & 0 & $0.06 / 4$ & 0 & 1 & 2 & $8 />256$ \\
\hline & & & $\geq 8$ & 3 & 1 & 0 & 0 & 1 & 1 & $4 / 8$ & 0 & 1 & 2 & $>256 />256$ \\
\hline
\end{tabular}

MIC, minimum inhibitory concentration; TEL, telithromycin; ERY, erythromycin; MIC ${ }_{50} / M_{1} C_{90}$, the MIC values for $50 \%$ or $90 \%$ of bacterial growth inhibition.

TABLE 3 | In vitro activity of TEL against $E$. faecalis isolates with ERY-specific resistant genes.

\begin{tabular}{|c|c|c|c|c|c|c|c|c|c|c|c|c|}
\hline \multicolumn{2}{|c|}{$\begin{array}{l}\text { Erythromycin } \\
\text { resistance genes }\end{array}$} & \multirow[t]{2}{*}{ No. (\%) } & \multicolumn{6}{|c|}{ TEL MIC ( $\mu \mathrm{g} / \mathrm{mL})$} & \multicolumn{4}{|c|}{ ERY MIC $(\mu \mathrm{g} / \mathrm{mL})$} \\
\hline & & & $\leq 0.5$ & 1 & 2 & 4 & $\geq 8$ & $\mathrm{MIC}_{50} / \mathrm{MIC}_{90}$ & $\leq 0.5$ & $1-4$ & $\geq 8$ & $\mathrm{MIC}_{50} / \mathrm{MIC}_{90}$ \\
\hline Total & & 280 & 116 & 7 & 27 & 97 & 33 & $2 / 8$ & 10 & 58 & 212 & $>256 />256$ \\
\hline \multirow[t]{2}{*}{ ermA } & + & $11(3.9)$ & 1 & 0 & 0 & 6 & 4 & $4 / 8$ & 0 & 0 & 11 & $>256 />256$ \\
\hline & - & $269(96.1)$ & 115 & 7 & 27 & 91 & 29 & $2 / 8$ & 10 & 58 & 201 & $>256 />256$ \\
\hline \multirow[t]{2}{*}{ ermB } & + & $188(67.1)$ & 39 & 4 & 23 & 92 & 30 & $4 / 8$ & 1 & 8 & 179 & $>256 />256$ \\
\hline & - & $92(32.9)$ & 77 & 3 & 4 & 5 & 3 & $0.06 / 2$ & 9 & 50 & 33 & $2 />256$ \\
\hline \multirow[t]{2}{*}{ ermC } & + & $0(0)$ & 0 & 0 & 0 & 0 & 0 & - & 0 & 0 & 0 & - \\
\hline & - & $280(100)$ & 116 & 7 & 27 & 97 & 33 & $2 / 8$ & 10 & 58 & 212 & $>256 />256$ \\
\hline
\end{tabular}

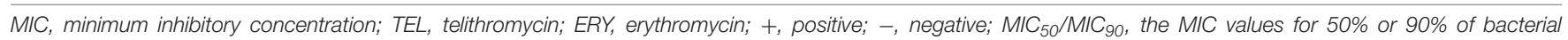
growth inhibition.

\section{DISCUSSION}

Enterococci species are commensal bacteria in the human gastrointestinal tract with the ability to cause various nosocomial infections. They not only have multiple inherent antimicrobial resistances, but are also able to acquire mutations and/or new resistance genes (Cetinkaya et al., 2000; Raza et al., 2018). Telithromycin, a novel ketolide antimicrobial agent, can be utilized for the treatment of respiratory infections. It retains its activity against most macrolide-resistant strains of Streptococcus pneumoniae and Streptococcus pyogenes (Spiers and Zervos, 2004; Wolter et al., 2008; Togami et al., 2009; Uzun et al., 2014). In addition, the efficacy of telithromycin against Enterococcus has been studied worldwide (Baltch et al., 2001; Bonnefoy et al., 2001; Mensa et al., 2003; Min et al., 2011), and it has been proven to be more effective against enterococci 
TABLE 4 | In vitro activity of TEL against E. faecium isolates with ERY-specific resistant genes.

\begin{tabular}{|c|c|c|c|c|c|c|c|c|c|c|c|c|}
\hline \multirow{2}{*}{\multicolumn{2}{|c|}{$\begin{array}{l}\text { Erythromycin } \\
\text { resistance genes }\end{array}$}} & \multirow[t]{2}{*}{ No. (\%) } & \multicolumn{6}{|c|}{ TEL MIC $(\mu \mathrm{g} / \mathrm{mL})$} & \multicolumn{4}{|c|}{ ERY MIC ( $\mu \mathrm{g} / \mathrm{mL})$} \\
\hline & & & \multirow{2}{*}{$\begin{array}{r}\leq 0.5 \\
25\end{array}$} & \multirow{2}{*}{$\begin{array}{l}1 \\
2\end{array}$} & \multirow{2}{*}{$\begin{array}{l}2 \\
12\end{array}$} & \multirow{2}{*}{$\begin{array}{c}4 \\
34\end{array}$} & \multirow{2}{*}{$\begin{array}{l}\geq 8 \\
49\end{array}$} & \multirow{2}{*}{$\frac{\mathbf{M I C}_{50} / \mathbf{M I C}_{90}}{4 / 8}$} & \multirow{2}{*}{$\begin{array}{c}\leq 0.5 \\
6\end{array}$} & \multirow{2}{*}{$\begin{array}{c}\mathbf{1 - 4} \\
12\end{array}$} & \multirow{2}{*}{$\begin{array}{l}\geq 8 \\
104\end{array}$} & \multirow{2}{*}{$\begin{array}{c}\mathbf{M I C}_{50} / \mathbf{M I C}_{90} \\
\geq 0.25-256\end{array}$} \\
\hline Total & & 122 & & & & & & & & & & \\
\hline \multirow[t]{2}{*}{ ermA } & + & $12(9.8)$ & 2 & 0 & 2 & 3 & 5 & $4 / 8$ & 0 & 2 & 10 & $128 />256$ \\
\hline & - & $110(90.2)$ & 23 & 2 & 10 & 31 & 44 & $4 / 8$ & 6 & 10 & 94 & $>128 />256$ \\
\hline \multirow[t]{2}{*}{ ermB } & + & $40(32.8)$ & 2 & 0 & 3 & 21 & 14 & $4 / 8$ & 0 & 2 & 38 & $128 />256$ \\
\hline & - & $82(67.2)$ & 23 & 2 & 9 & 13 & 35 & $4 / 8$ & 6 & 10 & 66 & $>128 />256$ \\
\hline \multirow[t]{2}{*}{ ermC } & + & $56(45.9)$ & 5 & 2 & 7 & 10 & 32 & $8 / 8$ & 0 & 1 & 55 & $>256 />256$ \\
\hline & - & $66(54.1)$ & 20 & 0 & 5 & 24 & 17 & $4 / 8$ & 6 & 11 & 49 & $>128 />256$ \\
\hline
\end{tabular}

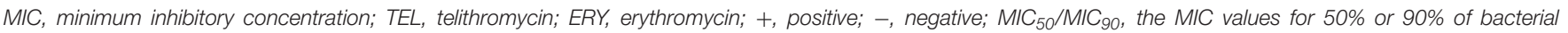
growth inhibition.

TABLE 5 | TEL MIC values of predominant STs in E. faecalis isolates with the ermB gene.

\begin{tabular}{|c|c|c|c|c|c|c|c|c|c|c|c|c|}
\hline \multirow[t]{2}{*}{ ST } & \multirow[t]{2}{*}{ No. (\%) } & \multicolumn{6}{|c|}{ TEL MIC ( $\mu \mathrm{g} / \mathrm{mL})$} & \multicolumn{4}{|c|}{ ERY MIC ( $\mu \mathrm{g} / \mathrm{mL})$} & \multirow[t]{2}{*}{ ermB } \\
\hline & & $\leq 0.5$ & 1 & 2 & 4 & $\geq 8$ & $\mathrm{MIC}_{50} / \mathrm{MIC}_{90}$ & $\leq 0.5$ & $1-4$ & $\geq 8$ & $\mathrm{MIC}_{50} / \mathrm{MIC}_{90}$ & \\
\hline ST16 & $78(27.9)$ & 18 & 3 & 9 & 36 & 12 & $4 / 8$ & 0 & 6 & 72 & $>256 />256$ & 67 \\
\hline ST30 & $8(2.9)$ & 7 & 0 & 1 & 0 & 0 & $0.125 / 2$ & 0 & 2 & 6 & $128 />256$ & 6 \\
\hline ST179 & $72(25.7)$ & 14 & 0 & 5 & 39 & 14 & $4 / 8$ & 2 & 7 & 63 & $>256 />256$ & 62 \\
\hline
\end{tabular}

MIC, minimum inhibitory concentration; TEL, telithromycin; ERY, erythromycin; MIC $50 / M I C_{90}$, the MIC values for $50 \%$ or $90 \%$ of bacterial growth inhibition.

TABLE 6 | TEL MIC values of predominant STs in E. faecium isolates carrying the ermB and ermC genes.

\begin{tabular}{|c|c|c|c|c|c|c|c|c|c|c|c|c|c|}
\hline \multirow[t]{2}{*}{ ST } & \multirow[t]{2}{*}{ No. (\%) } & \multicolumn{6}{|c|}{ TEL MIC ( $\mu \mathrm{g} / \mathrm{mL})$} & \multicolumn{4}{|c|}{ ERY MIC ( $\mu \mathrm{g} / \mathrm{mL})$} & \multirow[t]{2}{*}{ ermB } & \multirow[t]{2}{*}{ ermC } \\
\hline & & $\leq 0.5$ & 1 & 2 & 4 & $\geq 8$ & $\mathrm{MIC}_{50} / \mathrm{MIC}_{90}$ & $\leq 0.5$ & $1-4$ & $\geq 8$ & $\mathrm{MIC}_{50} / \mathrm{MIC}_{90}$ & & \\
\hline ST18 & 18 (12.3) & 3 & 0 & 0 & 0 & 15 & $8 / 8$ & 0 & 1 & 17 & $>256 />256$ & 1 & 17 \\
\hline ST78 & $26(21.3)$ & 4 & 1 & 0 & 9 & 12 & $4 / 8$ & 3 & 1 & 22 & $128 />256$ & 16 & 3 \\
\hline ST80 & $7(5.7)$ & 0 & 0 & 1 & 3 & 3 & $4 / 8$ & 0 & 0 & 7 & $>128 />128$ & 4 & 0 \\
\hline
\end{tabular}

MIC, minimum inhibitory concentration; TEL, telithromycin; ERY, erythromycin; MIC $50 / M I C_{90}$, the MIC values for $50 \%$ or $90 \%$ of bacterial growth inhibition.

than some of first- and second-generation macrolides. For instance, telithromycin was found to be more potent against enterococci than erythromycin in a mouse peritonitis model (Singh et al., 2000). The $\mathrm{MIC}_{50} / \mathrm{MIC}_{90}$ of telithromycin were $8 / 32 \mu \mathrm{g} / \mathrm{mL}$ against vancomycin-resistance enterococci strains, whereas erythromycin and clarithromycin were completely inactive (Hamilton-Miller and Shah, 1998). In this study, the $\mathrm{MIC}_{50} / \mathrm{MIC}_{90}$ of ERY were $>256 />256 \mu \mathrm{g} / \mathrm{mL}$, and the resistance rate of ERY reached more than $75 \%$ in both $E$. faecalis and E. faecium isolates (Tables 1, 2). However, the $\mathrm{MIC}_{50} / \mathrm{MIC}_{90}$ of telithromycin in E. faecalis and E. faecium isolates were found to be as low as $2 / 8$ and $4 / 8 \mu \mathrm{g} / \mathrm{mL}$, respectively (Tables 1,2 ). This study thus delineated additional evidence to support the fact that telithromycin could be a promising antimicrobial drug for use against enterococci. Of note, the majority of clinical E. faecalis isolates remain susceptible to $\beta$-lactams which are inhibitors of cell wall synthesis, and time-kill curve studies demonstrated that telithromycin $(4 \times \mathrm{MIC})$ combined with ampicillin $(4 \times \mathrm{MIC})$ could kill more than 100 times more planktonic cells in comparison to telithromycin or ampicillin alone in two E. faecalis isolates (Supplementary Figures 2A,B), suggesting that the combination of telithromycin and ampicillin could improve bactericidal activities against $E$. faecalis.

Ever since erythromycin has been clinically applied, the MLSB resistance phenotype has been widely found in erythromycinresistant isolates of many bacteria species. For instance, Weisblum B et al. demonstrated that erythromycin-resistant $S$. aureus was related to the MLSB resistance phenotype after the clinical application of erythromycin (Weisblum and Demohn, 1969). This MLSB phenotype was determined by the erm genes producing erythromycin-resistant methylases, which induced methylation at specific adenosine residues on the $23 \mathrm{~S}$ rRNA, thus leading to the resistance of the newly synthesized ribosome to MLSB antibiotics (Westh et al., 1995; Ackermann and Rodloff, 2003). Previous studies have described that the emergence of high-level erythromycin resistance narrowed the clinical application of macrolides and their application might result in a poor prognosis, more severe recurrence, and higher mortality for the treatment of bacterial infections (Min et al., 2011; Celik et al., 2014). While the chemical structure of telithromycin is derived from erythromycin, telithromycin has a low potential for drug-drug interaction, and is less likely to induce MLSB 

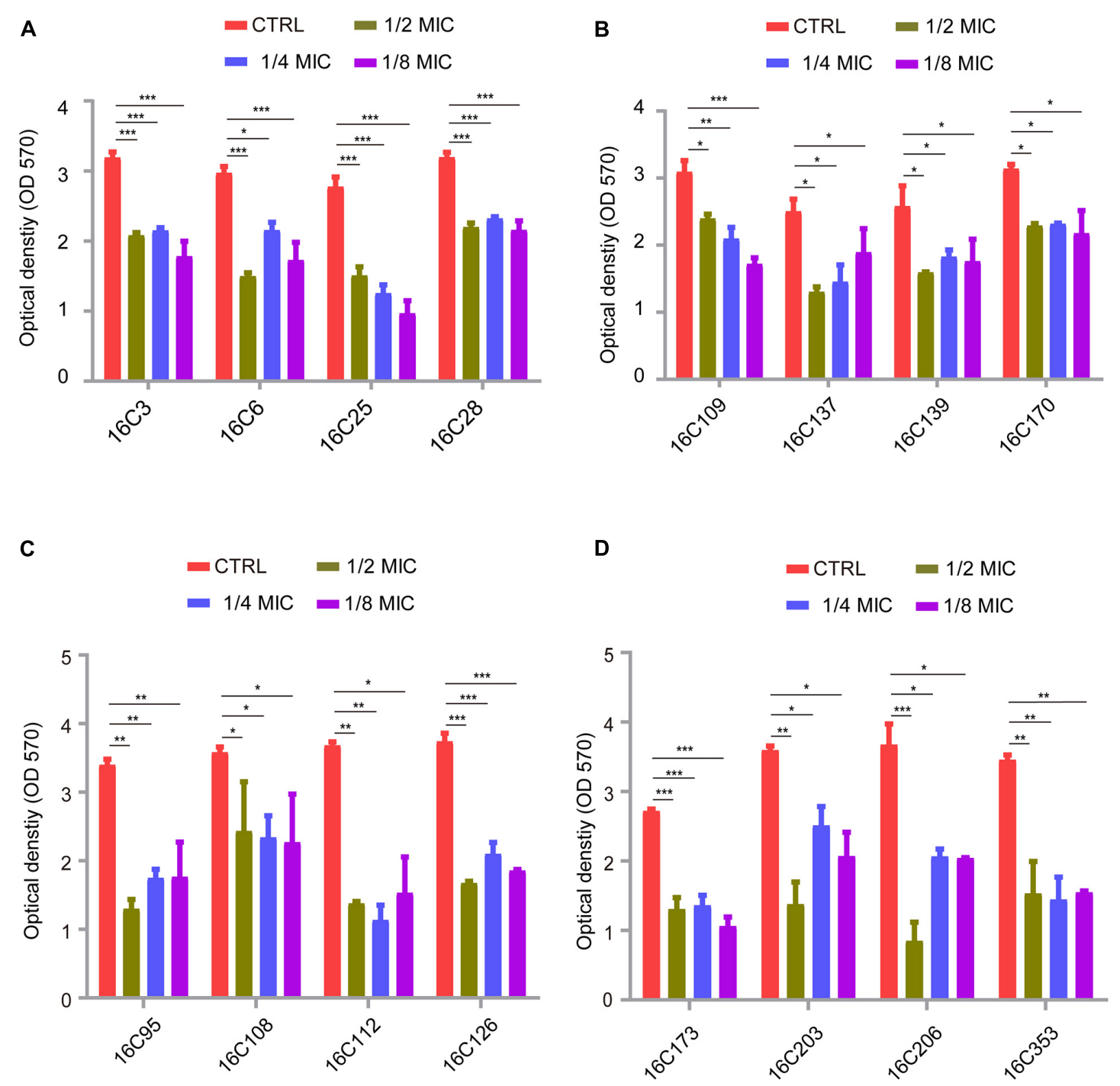

FIGURE 1 | Telithromycin inhibiting biofilm formation of $16 \mathrm{E}$. faecalis isolates. (A,B) The four isolates per group were treated with TEL at $1 / 2 \times$, $1 / 4 \times$, and $1 / 8 \times$ MICs, respectively. All of the eight isolates with the TEL MIC at $8 \mu \mathrm{g} / \mathrm{mL}$. (C,D) The eight isolates of the two groups were treated with TEL at $1 / 2 \times, 1 / 4 \times$, and $1 / 8 \times$ MICs. The MICs were 0.25, 0.5, 0.125, 0.125, 0.5, 0.125, 0.25, and 0.25 $\mu \mathrm{g} / \mathrm{mL}$, respectively. TSBG without antimicrobials was used as an untreated control. The data were presented as the mean $\pm \operatorname{SEM}\left(n=3\right.$ experiments), one-way analysis of variance (ANOVA), and a post hoc-Dunnett test, ${ }^{*} p<0.05$, ${ }^{* *} p<0.01$, ${ }^{* * *} p<0.001$, compared with the CTRL group.

resistance than macrolides with a 14- or 15-member ring (Benes, 2004). In this study, the ermA and ermB genes were found in ERY-resistant isolates of E. faecalis, and ermA, ermB, and erm $C$ genes were detected in E. faecium isolates (Tables 3, 4). However, telithromycin remained active against enterococci with erm genes. Recombination is involved in the genetic variation of resistance and virulence determinants, which might promote the hospital adaptation of Enterococcus bacteria such as E. faecalis and E. faecium (Homan et al., 2002; Ruiz-Garbajosa et al., 2006). In this study, E. faecalis isolates were grouped into 44 distinct STs, with the predominant STs being ST16 and ST179, which belonged to the clonal complex (CC16) (Bai et al., 2018). In addition, 25 STs were determined in E. faecium isolates, among which ST18 and ST78 were the main positive STs (Tables 5, 6). These results further confirmed that the presence of erm genes had minimal effects on the sensitivity of the predominant ST strains to telithromycin, suggesting its potential application for the treatment of some multi-resistant enterococci infections.

Evidence continues to accrue documenting the crucial role of biofilm formation in enterococcal infection (Ch'ng et al., 2019). The majority of clinical E. faecalis isolates are capable of forming biofilms on inanimate and living surfaces, which may promote antibiotic tolerance and reduce susceptibility to environmental influences or antimicrobial pressures (Paganelli et al., 2012; Holmberg and Rasmussen, 2016). Thus, targeting the biofilm formation of $E$. faecalis may potentially contribute to the treatment of enterococcal infections. As previously described, telithromycin could be considered as a novel inhibitor of 


$$
\text { A } \begin{aligned}
& \mathrm{CTRL}=\mathrm{AMP} \\
& =\mathrm{LZD}=\mathrm{TAN}
\end{aligned}
$$

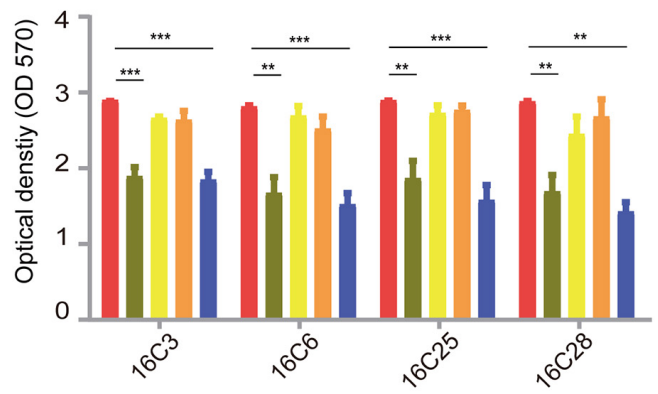

C

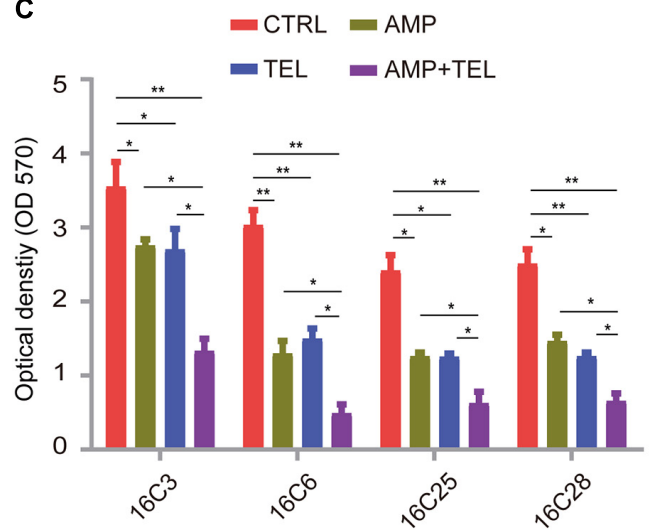

E

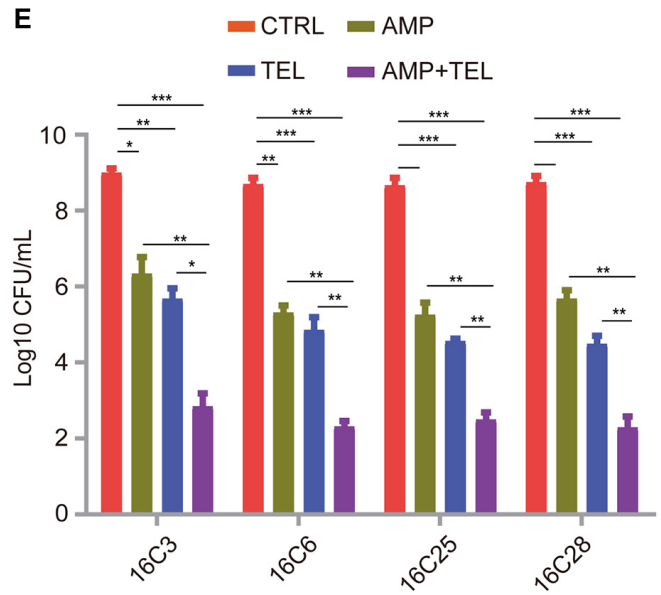

$\begin{aligned} & =\mathrm{CTRL} \\ & =\mathrm{AMP}\end{aligned}$

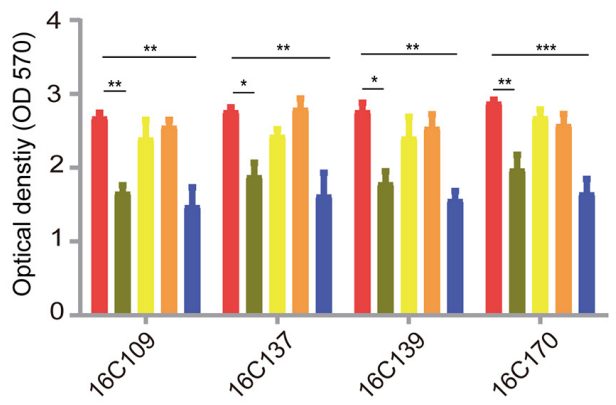

D

$$
=\mathrm{CTRL}=\mathrm{AMP}
$$

-TEL $=\mathrm{AMP}+\mathrm{TEL}$

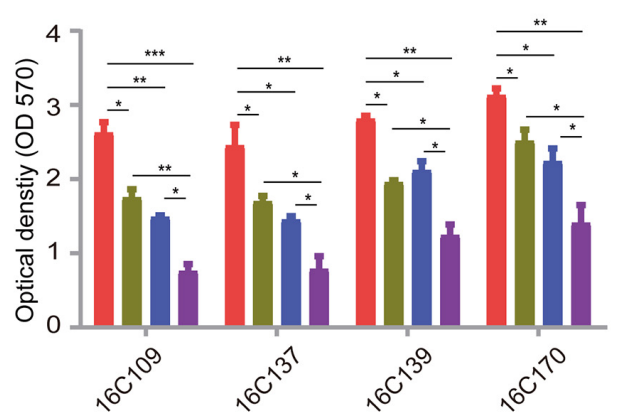

$\mathbf{F}$

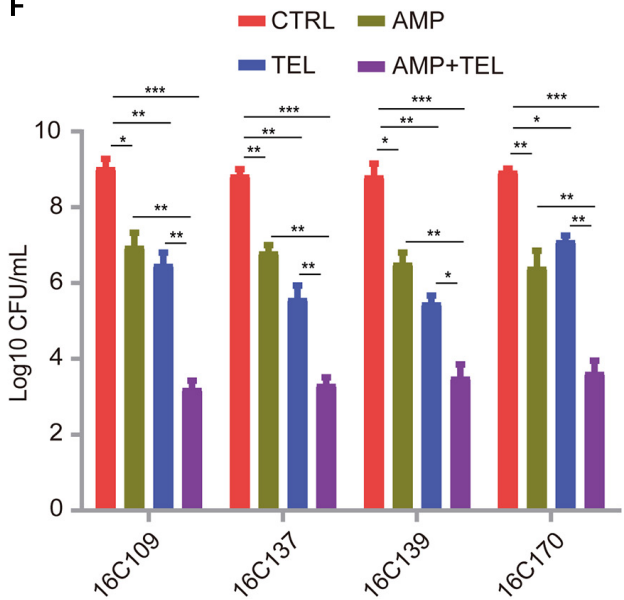

FIGURE 2 | Telithromycin alone or combined with ampicillin eradicating the established biofilms of eight $E$. faecalis isolates. (A,B) A total of eight isolates formed mature biofilms for $24 \mathrm{~h}$, then treated with AMP, VAN, LZD, or TEL at $8 \times$ MICs for $48 \mathrm{~h}$. The MICs of AMP, VAN, and TEL for these isolates were 2,1 , and $8 \mu \mathrm{g} / \mathrm{mL}$, respectively. The MICs of LZD for 16C3, 16C6, 16C25, and 16C28 isolates were $4 \mu \mathrm{g} / \mathrm{mL}$, the MICs for 16C109, 16C137, 16C139, and 16C170 were $2 \mu \mathrm{g} / \mathrm{mL}$. (C,D) A total of eight isolates formed mature biofilms for $24 \mathrm{~h}$, then were treated with AMP and TEL alone or TEL combined with AMP at $8 \times$ MICs for $48 \mathrm{~h}$. The MICs of AMP and TEL for these isolates were 2 and $8 \mu \mathrm{g} / \mathrm{mL}$, respectively. (E,F) A total of eight isolates formed mature biofilms for $24 \mathrm{~h}$, then were treated with AMP and TEL alone or TEL combined with AMP at $8 \times$ MICs for $48 \mathrm{~h}$. Then the adherent cells in these established biofilms were detected by the CFU numbers. The MICs of AMP and TEL for these isolates were 2 and $8 \mu \mathrm{g} / \mathrm{mL}$, respectively. TSBG without antimicrobials was used as an untreated control throughout. The data were presented as the mean \pm SEM ( $n=3$ experiments), one-way analysis of variance (ANOVA), and post hoc-Dunnett test, ${ }^{*} p<0.05,{ }^{* \star} p<0.01$, ${ }^{* \star *} p<0.001$, compared with the CTRL group.

S. aureus biofilms, which may be a result of the decreased expression of biofilm formation-related genes (Woo et al., 2017; Zheng et al., 2020), but there are no available data on the contribution of telithromycin susceptibility to biofilm phenotype in E. faecalis. The present study firstly showed that subinhibitory concentrations of telithromycin could inhibit the 
biofilm formation of $16 \mathrm{E}$. faecalis isolates (Figure 1). However, the data regarding biofilm formation inhibition does not show a dose-dependent effect. Therefore, further investigation is needed to characterize the inhibitory effect of telithromycin on the biofilm formation of E. faecalis.

Because biofilm prevention is not always possible, the removal of pre-existing enterococcal biofilms remains a necessity. Although the antibiofilm activity of antibiotics is likely hampered by poor penetration or slowed cell division in metabolically dormant biofilms, the use of antibiotics as first-line treatment for biofilm-associated infections is commonplace (Stewart, 2002). For example, the most recommended antibiotic treatment of endocarditis caused by E. faecalis involves ampicillin combined with gentamicin for 4-6 weeks (Habib et al., 2015). In this study, both telithromycin and ampicillin exhibited promising biofilminhibiting activity (40\% reduction) in E. faecalis (Figures 2A,B), these results are consistent with those previously reported in the in vitro activities of telithromycin or ampicillin against $S$. aureus or E. faecalis biofilms (Di Domenico et al., 2019; Zheng et al., 2020). Notably, treatment with an $8 \times$ MIC telithromycin/ampicillin combination exhibited a visible reduction $(\sim 70 \%)$ in mature E. faecalis biofilms (Figures 2C,D) and killed $70 \%$ of adherent cells in the established biofilms (Figures 2E,F). Therefore, the combination of telithromycin with ampicillin as an anti-adherence or antibiofilm strategy appears to be much more promising for the treatment of biofilm-associated enterococcal infections.

Accumulating evidence has revealed that there is a lower likelihood of resistance developing through the clinical use of telithromycin. For example, spontaneous resistance to telithromycin was at a low frequency in vitro. Telithromycin does not induce MLSB resistance and it shows low potential in selecting resistance or cross-resistance (Felmingham, 2001). In addition, pharmacodynamic studies suggested that telithromycin was generally well tolerated and a once-daily $800 \mathrm{mg}$ oral dose of telithromycin maintains an effective concentration in plasma for the treatment of respiratory tract infections involving the key respiratory pathogens (Drusano, 2001; Namour et al., 2001). However, information regarding kill kinetics and post-antibiotic effects for telithromycin against enterococci is limited. Thereafter, there is inadequate clinical evidence to suggest an optimal dosage regimen for telithromycin against enterococci. The present study demonstrated the antimicrobial susceptibility and antibiofilm activity of telithromycin against Enterococcus isolates in vitro, suggesting that this compound might be useful, alone or in combination, in some difficult to treat enterococcal infections. The further results of in vivo studies should provide some evidence to support such a possibility.

\section{CONCLUSION}

In summary, this study presents the effective antimicrobial activity of telithromycin against clinical enterococci isolates from China in comparison to that of ERY. Importantly, this study further demonstrated that telithromycin could inhibit the biofilm formation of E. faecalis and that telithromycin combined with ampicillin resulted in enhanced antimicrobial and antibiofilm activity. To our knowledge, this is the first study to present new insight into the antibiofilm activity of telithromycin against enterococci and provide further evidence for the potential clinical application of telithromycin/ampicillin combination for the treatment of Enterococcus infections.

\section{DATA AVAILABILITY STATEMENT}

The original contributions presented in the study are included in the article/Supplementary Material, further inquiries can be directed to the corresponding authors.

\section{AUTHOR CONTRIBUTIONS}

YX conducted the PCR analyses, MLST analysis, biofilms inhibition and eradication of E. faecalis, and drafted the manuscript. JC collected the bacterial isolates, performed the antimicrobials susceptibility tests, and gene manipulation. XS participated in gene manipulation, MLST analysis, and the inhibition of E. faecalis biofilms. GX and PL participated in the collection of bacterial isolates, MLST analysis, and the inhibition and eradication of E. faecalis biofilms. ZY and QD participated in the collection of bacterial isolates, and the inhibition and eradication of E. faecalis biofilms. ZC and JZ designed the study, analyzed the experimental data, and revised the manuscript. All authors have read and approved the manuscript.

\section{FUNDING}

This work was supported by the following grants: the collection of bacterial isolates, antimicrobials susceptibility tests, PCR analyses, and MLST analysis were supported by the Science, Technology and Innovation Commission of Shenzhen Municipality of Key Funds (JCYJ20180508162403996) and basic research funds (JCYJ20180302144721183, JCYJ20180302144340004, JCYJ20180302144345028, and JCYJ20180302144431923). The inhibition and eradication of E. faecalis biofilms were supported by the Sanming Project of Medicine in Shenzhen (No. SMGC201705029) and the Shenzhen Nanshan District Scientific Research Program of the People's Republic of China (Nos. 2019042, 2019051, 2019046, 2019027, and 2019040); provincial medical fund of Guangdong (2018116164215307), and Shenzhen Key Medical Discipline Construction Fund (No. SZXK06162).

\section{ACKNOWLEDGMENTS}

The authors would like to thank Yang Wu (Key Laboratory of Medical Molecular Virology of Ministries of Education and Health, School of Basic Medical Science and Institutes of Biomedical Sciences, Shanghai Medical College, Fudan University) for his excellent technical support and suggestions. 
The authors would also like to thank Ms. Cynthia Brast (University of Florida, Gainesville, FL, United States) for reviewing the manuscript. This manuscript has been released as a pre-print at ResearchSquare https://www.researchsquare.com/ article/rs-24213/v1 (Yanpeng et al., 2020).

\section{REFERENCES}

Ackermann, G., and Rodloff, A. C. (2003). Drugs of the 21st century: telithromycin (HMR 3647)-the first ketolide. J. Antimicrob. Chemother. 51, 497-511. doi: $10.1093 / \mathrm{jac} / \mathrm{dkg} 123$

Arias, C. A., and Murray, B. E. (2012). The rise of the Enterococcus: beyond vancomycin resistance. Nat. Rev. Microbiol. 10, 266-278. doi: 10.1038/ nrmicro2761

Bai, B., Hu, K., Li, H., Yao, W., Li, D., Chen, Z., et al. (2018). Effect of tedizolid on clinical Enterococcus isolates: in vitro activity, distribution of virulence factor, resistance genes and multilocus sequence typing. FEMS Microbiol. Lett. 3:365. doi: 10.1093/femsle/fnx284

Baldassarri, L., Cecchini, R., Bertuccini, L., Ammendolia, M. G., Iosi, F., Arciola, C. R., et al. (2001). Enterococcus spp. produces slime and survives in rat peritoneal macrophages. Med. Microbiol. Immunol. 190, 113-120. doi: 10.1007/ s00430-001-009698

Baltch, A. L., Smith, R. P., Ritz, W. J., and Bopp, L. H. (2001). Inhibitory and bactericidal effects of telithromycin (HMR 3647, RU 56647) and five comparative antibiotics, used singly and in combination, against vancomycinresistant and vancomycin-susceptible enterococci. Chemotherapy 47, 250-260. doi: $10.1159 / 000048531$

Benes, J. (2004). Telithromycin. Klin. Mikrobiol. Infekc. Lek. 10, 16-21.

Bonnefoy, A., Guitton, M., Delachaume, C., Le Priol, P., and Girard, A. M. (2001). In vivo efficacy of the new ketolide telithromycin (HMR 3647) in murine infection models. Antimicrob. Agents Chemother. 45, 1688-1692. doi: 10.1128/ aac.45.6.1688-1692.2001

Celik, S., Cakirlar, F. K., and Torun, M. M. (2014). Presence of vancomycin, aminoglycosides, and erythromycin resistance genes in enterococci isolated from clinical samples in Turkey. Clin. Lab. 60, 1801-1806. doi: 10.7754/clin. lab.2014.140211

Cetinkaya, Y., Falk, P., and Mayhall, C. G. (2000). Vancomycin-resistant enterococci. Clin. Microbiol. Rev. 13, 686-707. doi: 10.1128/cmr.13.4.686-707. 2000

Ch'ng, J. H., Chong, K. K. L., Lam, L. N., Wong, J. J., and Kline, K. A. (2019). Biofilm-associated infection by enterococci. Nat. Rev. Microbiol. 17, 82-94. doi: 10.1038/s41579-018-0107-Z

Costerton, J. W., Stewart, P. S., and Greenberg, E. P. (1999). Bacterial biofilms: a common cause of persistent infections. Science 284, 1318-1322. doi: 10.1126/ science. 284.5418 .1318

Darouiche, R. O. (2004). Treatment of infections associated with surgical implants. N. Engl. J. Med. 350, 1422-1429. doi: 10.1056/NEJMra035415

Di Domenico, E. G., Rimoldi, S. G., Cavallo, I., D’Agosto, G., Trento, E., Cagnoni, G., et al. (2019). Microbial biofilm correlates with an increased antibiotic tolerance and poor therapeutic outcome in infective endocarditis. BMC Microbiol. 19:228. doi: 10.1186/s12866-019-15961592

Di Rosa, R., Creti, R., Venditti, M., D’Amelio, R., Arciola, C. R., Montanaro, L., et al. (2006). Relationship between biofilm formation, the enterococcal surface protein (Esp) and gelatinase in clinical isolates of Enterococcus faecalis and Enterococcus faecium. FEMS Microbiol. Lett. 256, 145-150. doi: 10.1111/j.15746968.2006.00112.x

Douthwaite, S., and Champney, W. S. (2001). Structures of ketolides and macrolides determine their mode of interaction with the ribosomal target site. J. Antimicrob. Chemother. 48, 1-8. doi: 10.1093/jac/48.suppl_2.1

Drusano, G. (2001). Pharmacodynamic and pharmacokinetic considerations in antimicrobial selection: focus on telithromycin. Clin. Microbiol. Infect. 7, 24-29.

Duprè, I., Zanetti, S., Schito, A. M., Fadda, G., and Sechi, L. A. (2003). Incidence of virulence determinants in clinical Enterococcus faecium and Enterococcus faecalis isolates collected in Sardinia (Italy). J. Med. Microbiol. 52, 491-498. doi: $10.1099 /$ jmm. 0.050385030

\section{SUPPLEMENTARY MATERIAL}

The Supplementary Material for this article can be found online at: https://www.frontiersin.org/articles/10.3389/fmicb. 2020.616797/full\#supplementary-material

Eliopoulos, G. M. (1997). Vancomycin-resistant enterococci. Mechanism and clinical relevance. Infect. Dis. Clin. North Am. 11, 851-865. doi: 10.1016/s08915520(05)7039370397

Felmingham, D. (2001). Microbiological profile of telithromycin, the first ketolide antimicrobial. Clin. Microbiol. Infect. 7, 2-10.

Fiore, E., Van Tyne, D., and Gilmore, M. S. (2019). Pathogenicity of Enterococci. Microbiol. Spectr. 4:7. doi: 10.1128/microbiolspec.GPP3-00532018

Habib, G., Lancellotti, P., Antunes, M. J., Bongiorni, M. G., Casalta, J. P., Del Zotti, F., et al. (2015). 2015 ESC Guidelines for the management of infective endocarditis: The Task Force for the Management of Infective Endocarditis of the European Society of Cardiology (ESC). Eur. Heart J. 36, 3075-3128. doi: 10.1093/eurheartj/ehv319

Hamilton-Miller, J. M., and Shah, S. (1998). Comparative in-vitro activity of ketolide HMR 3647 and four macrolides against gram-positive cocci of known erythromycin susceptibility status. J. Antimicrob. Chemother. 41, 649-653. doi: 10.1093/jac/41.6.649

Hidron, A. I., Edwards, J. R., Patel, J., Horan, T. C., Sievert, D. M., Pollock, D. A., et al. (2008). NHSN annual update: antimicrobial-resistant pathogens associated with healthcare-associated infections: annual summary of data reported to the National Healthcare Safety Network at the Centers for Disease Control and Prevention, 2006-2007. Infect. Control. Hosp. Epidemiol. 29, 9961011. doi: $10.1086 / 591861$

Holmberg, A., and Rasmussen, M. (2016). Mature biofilms of Enterococcus faecalis and Enterococcus faecium are highly resistant to antibiotics. Diagn. Microbiol. Infect. Dis. 84, 19-21. doi: 10.1016/j.diagmicrobio.2015.09.012

Homan, W. L., Tribe, D., Poznanski, S., Li, M., Hogg, G., Spalburg, E., et al. (2002). Multilocus sequence typing scheme for Enterococcus faecium. J. Clin. Microbiol. 40, 1963-1971. doi: 10.1128/jcm.40.6.1963-1971.2002

Jakubovics, N. S., and Burgess, J. G. (2015). Extracellular DNA in oral microbial biofilms. Microbes Infect. 17, 531-537. doi: 10.1016/j.micinf.2015.03.015

Jett, B. D., Huycke, M. M., and Gilmore, M. S. (1994). Virulence of enterococci. Clin. Microbiol. Rev. 7, 462-478. doi: 10.1128/cmr.7.4.462

Jones, M. E., Draghi, D. C., Thornsberry, C., Karlowsky, J. A., Sahm, D. F., and Wenzel, R. P. (2004). Emerging resistance among bacterial pathogens in the intensive care unit-a European and North American Surveillance study (2000-2002). Ann. Clin. Microbiol. Antimicrob. 3:14. doi: 10.1186/1476-07 11-314

Lebeaux, D., Ghigo, J. M., and Beloin, C. (2014). Biofilm-related infections: bridging the gap between clinical management and fundamental aspects of recalcitrance toward antibiotics. Microbiol. Mol. Biol. Rev. 78, 510-543. doi: $10.1128 / \mathrm{mmbr} .0001314$

Lewis, K. (2001). Riddle of biofilm resistance. Antimicrob. Agents Chemother. 45, 999-1007. doi: 10.1128/aac.45.4.999-1007.2001

Marrie, T. J., Nelligan, J., and Costerton, J. W. (1982). A scanning and transmission electron microscopic study of an infected endocardial pacemaker lead. Circulation 66, 1339-1341. doi: 10.1161/01.cir.66.6.1339

Megran, D. W. (1992). Enterococcal endocarditis. Clin. Infect. Dis. 15, 63-71. doi: $10.1093 /$ clinids/15.1.63

Mendes, R. E., Castanheira, M., Farrell, D. J., Flamm, R. K., Sader, H. S., and Jones, R. N. (2016). Longitudinal (2001-14) analysis of enterococci and VRE causing invasive infections in European and US hospitals, including a contemporary (2010-13) analysis of oritavancin in vitro potency. J. Antimicrob. Chemother. 71, 3453-3458. doi: 10.1093/jac/dkw319

Menninger, J. R. (1995). Mechanism of inhibition of protein synthesis by macrolide and lincosamide antibiotics. J. Basic Clin. Physiol. Pharmacol. 6, 229-250. doi: 10.1515/jbcpp.1995.6.3-4.229

Mensa, J., García-Vázquez, E., and Vila, J. (2003). Macrolides, ketolides and streptogramins. Enferm. Infecc. Microbiol. Clin. 21:219.

Min, Y. H., Yoon, E. J., Kwon, A. R., Shim, M. J., and Choi, E. C. (2011). Alterations in regulatory regions of erm(B) genes from clinical isolates of 
enterococci resistant to telithromycin. Arch. Pharm. Res. 34, 2149-2154. doi: 10.1007/s12272-011-12191214

Mohamed, J. A., and Huang, D. B. (2007). Biofilm formation by enterococci. J. Med. Microbiol. 56, 1581-1588. doi: 10.1099/jmm.0.4733147330

Murray, B. E., and Weinstock, G. M. (1999). Enterococci: new aspects of an old organism. Proc. Assoc. Am. Physicians 111, 328-334. doi: 10.1046/j.1525-1381. 1999.99241.x

Namour, F., Wessels, D. H., Pascual, M. H., Reynolds, D., Sultan, E., and Lenfant, B. (2001). Pharmacokinetics of the new ketolide telithromycin (HMR 3647) administered in ascending single and multiple doses. Antimicrob. Agents Chemother. 45, 170-175. doi: 10.1128/aac.45.1.170-175.2001

Nguyen, M., and Chung, E. P. (2005). Telithromycin: the first ketolide antimicrobial. Clin. Ther. 27, 1144-1163. doi: 10.1016/j.clinthera.2005.08.009

Paganelli, F. L., Willems, R. J., and Leavis, H. L. (2012). Optimizing future treatment of enterococcal infections: attacking the biofilm? Trends Microbiol. 20, 40-49. doi: 10.1016/j.tim.2011.11.001

Raza, T., Ullah, S. R., Mehmood, K., and Andleeb, S. (2018). Vancomycin resistant Enterococci: A brief review. J. Pak. Med. Assoc. 68, 768-772.

Richards, M. J., Edwards, J. R., Culver, D. H., and Gaynes, R. P. (2000). Nosocomial infections in combined medical-surgical intensive care units in the United States. Infect. Control. Hosp. Epidemiol. 21, 510-515. doi: 10.1086/ 501795

Ruiz-Garbajosa, P., Bonten, M. J., Robinson, D. A., Top, J., Nallapareddy, S. R., Torres, C., et al. (2006). Multilocus sequence typing scheme for Enterococcus faecalis reveals hospital-adapted genetic complexes in a background of high rates of recombination. J. Clin. Microbiol. 44, 2220-2228. doi: 10.1128/jcm. 025962595

Sandoe, J. A. T., Witherden, I. R., Cove, J. H., Heritage, J., and Wilcox, M. H. (2003). Correlation between enterococcal biofilm formation in vitro and medicaldevice-related infection potential in vivo. J. Med. Microbiol. 52, 547-550. doi: 10.1099/jmm.0.052015200

Shoji, T., Takaya, A., Sato, Y., Kimura, S., Suzuki, T., and Yamamoto, T. (2015). RlmCD-mediated U747 methylation promotes efficient G748 methylation by methyltransferase RlmAII in 23S rRNA in Streptococcus pneumoniae; interplay between two rRNA methylations responsible for telithromycin susceptibility. Nucleic Acids Res. 43, 8964-8972. doi: 10.1093/nar/gkv609

Sievert, D. M., Ricks, P., Edwards, J. R., Schneider, A., Patel, J., Srinivasan, A., et al. (2013). Antimicrobial-resistant pathogens associated with healthcareassociated infections: summary of data reported to the National Healthcare Safety Network at the Centers for Disease Control and Prevention, 2009-2010. Infect. Control. Hosp. Epidemiol. 34, 1-14. doi: 10.1086/668770

Singh, K. V., Zscheck, K. K., and Murray, B. E. (2000). Efficacy of telithromycin (HMR 3647) against enterococci in a mouse peritonitis model. Antimicrob. Agents Chemother. 44, 3434-3437. doi: 10.1128/aac.44.12.3434-3437.2000

Spiers, K. M., and Zervos, M. J. (2004). Telithromycin. Expert Rev. Anti. Infect. Ther. 2, 685-693. doi: 10.1586/14789072.2.5.685

Stewart, P. S. (2002). Mechanisms of antibiotic resistance in bacterial biofilms. Int. J. Med. Microbiol. 292, 107-113. doi: 10.1078/1438-42214196

Sun, X., Lin, Z. W., Hu, X. X., Yao, W. M., Bai, B., Wang, H. Y., et al. (2018). Biofilm formation in erythromycin-resistant Staphylococcus aureus and the relationship with antimicrobial susceptibility and molecular characteristics. Microb. Pathog. 124, 47-53. doi: 10.1016/j.micpath.2018.08.021

Togami, K., Chono, S., Seki, T., and Morimoto, K. (2009). Distribution characteristics of telithromycin, a novel ketolide antimicrobial agent applied for treatment of respiratory infection, in lung epithelial lining fluid and alveolar macrophages. Drug Metab. Pharmacokinet 24, 411-417. doi: 10.2133/dmpk. 24.411

Uzun, B., Güngör, S., Pektaş, B., Aksoy Gökmen, A., Yula, E., Koçal, F., et al. (2014). [Macrolide-lincosamide-streptogramin B (MLSB) resistance phenotypes in clinical Staphylococcus isolates and investigation of telithromycin activity]. Mikrobiyol. Bul. 48, 469-476. doi: 10.5578/mb.7748

van Harten, R. M., Willems, R. J. L., Martin, N. I., and Hendrickx, A. P. A. (2017). Multidrug-Resistant Enterococcal Infections: New Compounds, Novel
Antimicrobial Therapies? Trends Microbiol. 25, 467-479. doi: 10.1016/j.tim. 2017.01.004

Van Laethem, Y., and Sternon, J. (2003). [Telithromycin, first ketolide]. Rev. Med/. Brux 24, 42-46.

Vester, B., and Douthwaite, S. (2001). Macrolide resistance conferred by base substitutions in 23S rRNA. Antimicrob. Agents Chemother. 45, 1-12. doi: 10. 1128/aac.45.1.1-12.2001

Wang, Y., Xiong, Y., Wang, Z., Zheng, J., Xu, G., Deng, Q., et al. (2020). Comparison of solithromycin with erythromycin in Enterococcus faecalis and Enterococcus faecium from China: antibacterial activity, clonality, resistance mechanism, and inhibition of biofilm formation. J. Antibiot. doi: 10.1038/ s41429-020-00374372 [Online ahead of print].

Weiner, L. M., Webb, A. K., Limbago, B., Dudeck, M. A., Patel, J., Kallen, A. J., et al. (2016). Antimicrobial-Resistant Pathogens Associated With HealthcareAssociated Infections: Summary of Data Reported to the National Healthcare Safety Network at the Centers for Disease Control and Prevention, 20112014. Infect. Control. Hosp. Epidemiol. 37, 1288-1301. doi: 10.1017/ice.20 16.174

Weisblum, B. (1995). Erythromycin resistance by ribosome modification. Antimicrob. Agents Chemother. 39, 577-585. doi: 10.1128/aac.39.3.577

Weisblum, B., and Demohn, V. (1969). Erythromycin-inducible resistance in Staphylococcus aureus: survey of antibiotic classes involved. J. Bacteriol. 98, 447-452. doi: 10.1128/jb.98.2.447-452.1969

Westh, H., Hougaard, D. M., Vuust, J., and Rosdahl, V. T. (1995). Prevalence of erm gene classes in erythromycin-resistant Staphylococcus aureus strains isolated between 1959 and 1988. Antimicrob. Agents Chemother. 39, 369-373. doi: 10.1128/aac.39.2.369

Wolter, N., Smith, A. M., Farrell, D. J., Northwood, J. B., Douthwaite, S., and Klugman, K. P. (2008). Telithromycin resistance in Streptococcus pneumoniae is conferred by a deletion in the leader sequence of erm(B) that increases rRNA methylation. Antimicrob. Agents Chemother. 52, 435-440. doi: 10.1128/ aac.010741077

Woo, S. G., Lee, S. Y., Lee, S. M., Lim, K. H., Ha, E. J., and Eom, Y. B. (2017). Activity of novel inhibitors of Staphylococcus aureus biofilms. Folia Microbiol. 62, 157-167. doi: 10.1007/s12223-016-0485484

Yanpeng, X., Junwen, C., Xiang, S., Guangjian, X., Peiyu, L., Qiwen, D., et al. (2020). In vitro antibacterial activity of telithromycin against Enterococcus spp. isolated from China. BMC Microbiol. 2020:24213. doi: 10.21203/rs.3.rs-24 213/v1

Zheng, J. X., Sun, X., Lin, Z. W., Qi, G. B., Tu, H. P., Wu, Y., et al. (2019). In vitro activities of daptomycin combined with fosfomycin or rifampin on planktonic and adherent linezolid-resistant isolates of Enterococcus faecalis. J. Med. Microbiol. 68, 493-502. doi: 10.1099/jmm.0.000945

Zheng, J. X., Tu, H. P., Sun, X., Xu, G. J., Chen, J. W., Deng, Q. W., et al. (2020). In vitro activities of telithromycin against Staphylococcus aureus biofilms compared with azithromycin, clindamycin, vancomycin and daptomycin. J. Med. Microbiol. 69, 120-131. doi: 10.1099/jmm.0.001122

Zheng, J. X., Wu, Y., Lin, Z. W., Pu, Z. Y., Yao, W. M., Chen, Z., et al. (2017). Characteristics of and Virulence Factors Associated with Biofilm Formation in Clinical Enterococcus faecalis Isolates in China. Front. Microbiol. 8:2338. doi: $10.3389 /$ fmicb. 2017.02338

Conflict of Interest: The authors declare that the research was conducted in the absence of any commercial or financial relationships that could be construed as a potential conflict of interest.

Copyright (c) 2021 Xiong, Chen, Sun, Xu, Li, Deng, Yu, Chen and Zheng. This is an open-access article distributed under the terms of the Creative Commons Attribution License (CC BY). The use, distribution or reproduction in other forums is permitted, provided the original author(s) and the copyright owner(s) are credited and that the original publication in this journal is cited, in accordance with accepted academic practice. No use, distribution or reproduction is permitted which does not comply with these terms. 